\title{
ROLE DEVELOPMENT OF NURSE MANAGERS IN THE CHANGING HEALTH CARE PRACTICE
}

\author{
Joel Rey U. Acob', Wiwin Martingsih ${ }^{2}$ \\ ${ }^{1}$ Faculty of Nursing, Visayas State University, Phillipines \\ ${ }^{2}$ Health Polytechnic of Malang, Indonesia \\ email: joel.acob@vsu.edu.ph,wiwin_martiningsih@yahoo.co.id
}

\begin{abstract}
Rapid changes in today's healthcare industry are reshaping nurses' role. The emergence of new healthcare system, the shift from service to business orientations, and extensive redesign of workplace affects the where and how nursing care is delivered as well as those who delivered the care. In the Philippines, healthcare system is in the midst of dramatic evolvement- the devolution of hospitals to LGUs, free healthcare for senior citizens and no balance billing policy yielding to increased client-nurse ratio. These impacts change the roles of nurse managers and their practice. The study aimed to understand the experience and phenomena of nurse managers, their roles in the dynamics of healthcare practice, and seek ways to enhance the development of these roles. The study used descriptive phenomenology-qualitative design, and utilized Colaizzi method during data analyses. Through researcher-made guide questions, the study employed purposively nurse managers as key informants from tertiary hospitals that experienced devolution using the inclusion and exclusion criteria. The researchers conducted interviews with audiotapes that then later transcribed. The study revealed that nurse managers encountered challenges in the workplace like deprivation of responsibility, less administrative support that challenged their responsibilities. The nurse managers identified new roles that they have developed over time like Carative-managerial role, responsibility to educate and the responsibility to the nursing profession. They are believers that the enhancement of such roles is realized through rest and recreation among staffs to avoid burnout and exhaustion, acquiring continuing professional education, clinical teaching and mentoring skills strategies to understand better human behavior. The researchers recommends consumption of such study findings as basis for improving hospital facilities for the provision of patient safety, revisiting and strengthening the hiring and screening policies for new nurses, administrative support for staff development and as basis to conduct further studies on the emerging roles to other settings.
\end{abstract}

Keywords: changing roles, nurses roles, nurse managers, emerging roles, health care practice, role development

\section{INTRODUCTION}

The changing health care system challenges the nurse managers of today. Whether they work on a medical floor in an acute care hospital or in a critical care unit, the nurse manager must deal with other people who work with them and for them, and they must use the resources wisely. A nurse manager on the other hand must recognize the need for growth within, which then translates into improvement of one's practice. Practicing nurse managers illustrate role perceptions; cite decision- making and problem solving as major roles for which maintaining objectivity is a special challenge.

Rapid changes in today's health care industry are reshaping the nurse's role. The emergence of new health care systems, the shift from service orientation to business orientation, and an extensive redesign of the workplace directly affect where and how nursing care is delivered as well as those who deliver the care.

To manage well, nurses must understand the health care system, the organizations they work and resources as well. They need to recognize what 
external factors affect their work and how to influence those forces. Nurse Managers must equip oneself to address the different situations concerning their roles.

In the Philippines, Health Care System is in the midst of significant and dramatic development as it continues to rapidly evolve - the devolution of hospitals to the Local Government Unit; free health care for the senior citizens; and the no balance billing policy for the indigents. These resulted to increase number of patients in the hospital, which in turn increased the workload of staff nurses and nurse to patient ratio adding more burdens to the nurse managers.

The impact of these changes greatly affects the role of nurse managers in their practice. They are tasked with a wider range of playing both the key to ensuring quality patient care and excellent workplace for staff nurses.

During the literature review, no study had been made to document how the nurse managers respond with dynamism to the changing health care practice. This study will deeply understand the phenomena of role development of nurse managers in the changing health care practice which must be looked into for possible policy development for the improvement of nursing practice.

\section{METHOD}

The study utilized qualitative phenomenology to help shape the nurse's perception of a problem or situation and their conceptualization of potential solutions (Streuber, 2003). Purposive sampling was utilized in selecting the key informants following the inclusion criteria. The researcher chose nurses with appointment as nurse managers in any area of the hospital as participants either male or female, 35 years old and above with at least five years continuous experience. The study was limited to nurses working in a tertiary government hospital in the provinces of Negros Oriental and Leyte, Philippines.

The researcher engaged in some activities with the participants such as morning rounds, endorsements, consultative meeting to enrich information on the actual daily transcations which involves nurse managers' decision-making. Each participant signed informed consent prior to the conduct of the study and data were collected through semi-structured interview. To establish trustworthiness and credibility of data, the researchers observed prolonged involvement, persistent observation and triangulation. Data were transcribed verbatim from the recorded audiotapes.

\section{RESULT}

Themes on the challenges of nurse managers in the modern day practice:

- Deprivation of responsibility

- Less administrative support

- Challenges to responsibilty

Development of themes on the new roles experienced by nurse managers:

- Collegial responsibility (carative managerial role)

- Responsibility to educate ( intermediary role)

- Responsibility to the nursing profession (instructor's role)

\section{DISCUSSION}

On challenges of nurse managers in the modern day practice. The nurse managers experienced challenges in their day-to-day practice. One nurse manager revealed that she was deprived with responsibility especially on abiding hospital policies and protocols. Staff nurses were sometimes not cooperative by not following duty schedules, which later lead to destruction of program and duty workloads of other staffs. As a nurse manager, they made it sure, that prior to the commencement of the next shift, receiving staffs should be complete atleast to continue the care. In the management, nurse managers do not only see human resource related to patient care (Towse, 2004); their scope expands to facilities and resources that were utilized during the entire process of patient caring. When the hospital lacks precautionary measures to ensure patient safety, the nurse managers perceived this problem since it affects the totality of human caring (ANSAP, 2001). On one hand, there are staff nurses who are adding burden or problem who in turn challenged the professional decision-making skills of the nurse managers. Majority of the informants agreed that instances like not following simple protocols in ward works, abseentism without prior notification of healthcare members and even conerns of safe workplace and environmental affairs contributes to disappointments of nurse managers. 
On new roles experienced and have developed by nurse mangers in the workplace. Collegial responsibility refers to actions of nurse managers in uplifting the professional maturity of staff nurses. This was evidenced when nurse managers uttered that millenial nurses as per experienced were quite passive in patient interaction. As immediate superiors, they made certain measures to maintain quality care amidst scarcity of resources through preceptorship (Masters, 2005), constant mentoring and shadowing as part of carative role to novice nurses. The nurse managers have to remind consistently and frequently new nurses about the standards of care practice because they are not compliant and abide less in it. The head nurse expressed feeling of being an instructor than a supervisor. One nurse manager spoke that by experience, she double-checked new nurses especially in terms of work performance and execution of tasks and deliverables to their patients, thus representing instructor's function. Head nurses should assume specific roles to have a more focused task for their human resource development. This includes giving of orientation to newly hired nurses on policies affecting their practice, and mentoring skills as well.

\section{CONCLUSION AND SUGGESTION}

\section{Conclusion}

The lived experiences of the participants in this study confirmed that nurse managers encountered various challenges in the workplace, which include deprivation of responsibility, less administrative support, which confront their responsibilities.

The nurse managers identified new roles that they have developed over time which include collegial responsibility, to educate young breed of healthcare workers and responsibility to the profession.

The new identified roles can be further enhanced through rest and recreation program among staffs to avoid burn out and exhaustion; assumption specific roles to have a more focused task for their human resource development such as giving of orientation to newly hired nurses on policies affecting their practice, and mentoring skills as well. Embodying knowledge and attitude in response to the changing work environment and people. This an be addressed through acquiring continuing professional education, clinical teaching strategies to appreciate better human behaviour.

\section{Suggestion}

From the findings of this study, the following recommendations are offered in relation to research, education, and practice to contribute for the enhancement of the phenomenon at hand.

The findings will be disseminated to the following:

Nurse managers so that findings may be translated into practice; and hospital administrators and chief nurses of the participating hospitals so their concerns will be given consideration.

The findings of this study will be the basis for:

Improving facilities in the hospital for the provision of patient safety. This can be made possible through proper requisition to the hospital administrators.

Revisiting and strengthening the hiring and screening policies for new nurses.

Supporting activities for staff development such as rest and recreation for employee and continuing Professional Education (CPE) to nurse mangers and staff nurses.

\section{REFERENCES}

Association of Nursing Service Administrators of the Philippines, Inc. 2001. Standards of Nursing Services.

Ereaut, G. (n.d.). What is Qualitative Research.Retrieved December 10, 2012 fromwww.qsinternatonal.com/ what-is-qualitative-research

Masters, Kathleen. 2005. Role development in Professional Nursing Practice 2nd ed. Jones \& Bartlett Publishers, Sudburg, Massachusets

Polit, D. \& Beck, C. 2004. Nursing research: Principles \& methods. (7th ed.). Lippincott Wiliams\& Wilkins, Philadelphia

Streubert, S. \& Carpenter, D. 2003. Qualitative research in nursing: Advancing the humanistic imperative 3rd ed. Lippincott Williams \& Wilkins, Philadelphia

Towse, A., Mills, A., \& Tangcharoensathien, V. 2004. Learning from Thailand's health reforms. doi: 10.1136/bmj.328.7431.103, 328(7431): 103-105

Oxford Business Group. 2016. The Philippines' health sector undergoing significant changes. Retrieved from https://www.oxfordbusinessgroup.com

Sanders, C. 2003. Application of Collaizzi's method: Interpretation of an auditable decision trail by a novice researcher. Contemporary Nurse 14: 292-302. 